\title{
Efecto vasodilatador e inhibidor de vasoconstricción del extracto hidroalcohólico de hojas de Olea europaea (olivo) sobre anillos aórticos de ratas
}

\author{
Vasodilator and vasoconstriction inhibitor effect of Olea europaea (olive) leaf
} hydroalcoholic extract on rat aortic rings

\author{
Job Nexar-QH ${ }^{1,2,3}$, Jhon Sillo-Surco ${ }^{1,2,3}$ \\ 'Estudiante de medicina, Universidad Nacional de San Agustín, Arequipa, Perú. \\ ${ }^{2}$ Centro de Investigación y Desarrollo Científico, Facultad de Medicina, Universidad Nacional de San Agustín, Arequipa, Perú. \\ ${ }^{3}$ Sociedad Cientifica de Estudiantes de Medicina Agustinos, Arequipa, Perú.
}

\begin{abstract}
Resumen
Objetivos: Evaluar el efecto vasodilatador, la inhibición de la vasoconstricción, así como el mecanismo responsable, del extracto hidroalcohólico de hojas de Olea europaea (olivo), sobre anillos aórticos en ratas. Diseño: Experimental. Lugar: Centro de Investigación y Desarrollo Científico, Facultad de Medicina, Universidad Nacional de San Agustín, Arequipa, Perú. Material biológico: Hojas de Olea europea y anillos aórticos de Rattus norvegicus, variedad albina swiss. Intervenciones: Se obtuvo un extracto hidroalcohólico de las hojas de Olea europaea. Se usó anillos aórticos de rata en cámara de órganos aislados y se registró la actividad vasomotora con un transductor de tensión isométrica. Se produjo contracción basal máxima con $\mathrm{CaCl}_{2} 6 \mathrm{mM}$ y se determinó el efecto vasodilatador con dosis de 25, 50 y $100 \mathrm{mg} / \mathrm{mL}$ de extracto hidroalcohólico de las hojas de Olea europaea. Se usó 1H-[1,2,4] oxadiazolo [4,3alquinoxalin-1-one] (ODQ) y nifedipino para determinar el mecanismo de acción. Se comparó la inhibición de la vasoconstricción tras la incubación durante 30 minutos con extracto $100 \mathrm{mg} / \mathrm{mL}$ y con captopril $10 \mu \mathrm{M}$. Principales medidas de resultados: Porcentaje de vasodilatación y de vasoconstricción. Resultados: Se obtuvo una vasodilatación de 7,20 $\pm 1,49 \%, 9,84 \pm 1,42 \%$ y 12,31 $\pm 1,16 \%$ para las dosis de 25, 50 y $100 \mathrm{mg} / \mathrm{mL}$, respectivamente, siendo significativa con la dosis de $100 \mathrm{mg} / \mathrm{mL}$. La vasodilatación se incrementó tras la administración de ODQ $100 \mathrm{mM}$. La vasodilatación se inhibió tras la incubación con ODQ $100 \mathrm{mM}$ más nifedipino $5 \mu \mathrm{M}$. No se encontró diferencia significativa entre la inhibición de la vasoconstricción con captopril $10 \mu \mathrm{M}$ y extracto a $100 \mathrm{mg} / \mathrm{mL}$. Conclusiones: El extracto hidroalcohólico de hojas de Olea europea, a una dosis de $100 \mathrm{mg} / \mathrm{mL}$, tiene efecto vasodilatador sobre anillos aórticos de ratas, mediado por el bloqueo de canales de calcio; además, posee efecto inhibidor de la vasoconstricción.

Palabras clave: Canales de calcio, extractos vegetales, hipertensión, Olea europaea, vasodilatación.
\end{abstract}

\section{Abstract}

Objectives: To determine the vasodilator and anti-vasoconstrictor effect of Olea europaea (olive) leaf hydroalcoholic extract on rat aortic rings and the mechanism involved. Design: Experimental. Location: Research and Scientific Development Center, Faculty of Medicine, Universidad Nacional de San Agustin, Arequipa, Peru. Biological material: Leaves of Olea europaea and aortic rings of Rattus norvegicus, swiss albina variety. Interventions: Hydroalcoholic extract was obtained from Olea europaea leaves. Rat aortic rings were placed in isolated organ chambers and the vasomotor activity was recorded with isometric tension transducer. Maximum basal contraction occurred at $\mathrm{CaCl}_{2}$, and $6 \mathrm{mM}$ vasodilator effect was determined at 25,50, and $100 \mathrm{mg} / \mathrm{mL}$ doses of the hydroalcoholic extract. $1 \mathrm{H}-[1,2,4]$ oxadiazole $[4,3$-alquinoxalin-1-one] $(\mathrm{ODQ})$ and nifedipine were used to determine the mechanism of action. We compared the inhibition of vasoconstriction after incubation for 30 minutes with the extract $100 \mathrm{mg} / \mathrm{mL}$ and with captopril $10 \mu \mathrm{M}$. Main outcome measures: Percentage of vasodilation and vasoconstriction. Results: There was vasodilatation of $7.20 \pm 1.49 \%, 9.84 \pm 1.42 \%$, and $12.31 \pm 1.16 \%$ for respectively 25,50 , and $100 \mathrm{mg} / \mathrm{mL}$ doses of hydroalcoholic extract; it was significant at doses of $100 \mathrm{mg} /$ $\mathrm{mL}$. Vasodilation increased after administration of ODQ $100 \mathrm{mM}$. Vasodilation was inhibited after incubation with ODQ $100 \mathrm{mM}$ plus nifedipine $5 \mu \mathrm{M}$. No significant difference was found between vasoconstriction inhibition with captopril $10 \mu \mathrm{M}$ or extract $100 \mathrm{mg} / \mathrm{mL}$. Conclusions: Olea europaea leaves hydroalcoholic extract at $100 \mathrm{mg} / \mathrm{mL}$ dose had calcium channel blockade-vasodilator effect and vasoconstriction inhibitory effect on rat aortic rings.

Keywords: Calcium channels, plant extracts, hypertension, Olea europaea, vasodilation.

An Fac med. 2013;74(4):285-90

\section{INTRODUCCIÓN}

El árbol del olivo (Olea europaea), que pertenece a la familia botánica de las Oleaceae, es un planta oriunda de la cuenca mediterránea. Actualmente, su distribución comprende tanto zonas de países europeos como otros países no europeos de clima parecido, como
Australia, Chile y Perú ${ }^{(1)}$. Sus frutos y el aceite obtenido son utilizados en la alimentación y adquiere un valor agregado debido a que el aceite y las hojas del olivo son utilizadas ampliamente en fitoterapia por sus importantes propiedades terapéuticas antimicrobianas, antivíricas, antihelmínticas, antifúngicas, hipoglicemiantes y diuréticas ${ }^{(2,3)}$.
El extracto e infusión de las hojas de olivo es valorado por los numerosos beneficios cardiovasculares, antioxidantes y actividad hipoglicemiante y diurética ${ }^{(2,3)}$. La composición es variada en las diferentes partes de la planta; las hojas contienen muchas sales minerales, ácidos grasos monoinsaturados, lípidos neutros, triterpenos y flavonoides ${ }^{(3,4)}$; 
además, contienen abundantes polifenoles, dentro de ellos los más importantes son hidroxitirosol o 3,4-dihidroxifeniletanol, oleuropeína (oleuropeósido) y oleurósido, sustancias con múltiples actividades biológicas a las que se atribuye sus bondades terapéuticas ${ }^{(1,5,6)}$.

Numerosos estudios concluyen que el aceite y las hojas de olivo presentan un efecto cardioprotector importante, resultado de su capacidad antioxidante, vasodilatadora, inhibición de la agregación plaquetaria y antiinflamatoria $(7,8)$, lo que hace reconocer al olivo y sus hojas como una de las alternativas naturales más difundidas para tratar múltiples enfermedades cardiovasculares, dentro de ellas la hipertensión arterial ${ }^{(9,10)}$. La hipertensión arterial es una enfermedad que continúa siendo un problema de salud pública en el Perú y cuya prevalencia en los últimos años fue aproximadamente $23,7 \%{ }^{(11)}$, cifra que no difiere en mucho con otros países de América Latina. Pese a los avances obtenidos en el tratamiento y prevención, se busca nuevas alternativas farmacológicas y fitoterapéuticas.

El efecto antihipertensivo de las hojas de olivo ha sido demostrado en estudios previos; así, la administración del extracto de las hojas por vía oral a ratas hipertensas logró reducir los niveles de presión sanguínea ${ }^{(12)}$. El mismo resultado se obtuvo al evaluar específicamente algunos compuestos aislados del extracto ${ }^{(13)}$. De igual manera, se ha evidenciado una reducción de los niveles elevados de presión sanguínea en aquellos pacientes a los que se les suministró extracto de hojas de olivo en diferentes dosis ${ }^{(14,15)}$. Sin embargo, pese a la evidencia científica existente sobre el efecto antihipertensivo del extracto de hoja de olivo y aunque se ha propuesto que dicho efecto antihipertensivo podría deberse a la acción vasodilatadora del extracto ${ }^{(16)}$, el mecanismo de acción no está aún esclarecido. Por tanto, es necesario determinar los mecanismos fisiológicos involucrados, lo que permitirá sentar las bases de futuros estudios orientados a obtener nuevas alternativas farmacológicas contra la hipertensión. Por otro lado, no existe evidencia científica sobre la acción vasodilatadora de las hojas de olivo procedente de cultivos del Perú, lo que también motiva nuestra investigación. Es por ello que el presente trabajo tiene como objetivo evaluar el efecto vasodilatador e inhibidor de vasoconstricción de las hojas de Olea europea y determinar el mecanismo fisiológico responsable de dicho efecto.

\section{MÉTODOS}

El diseño del trabajo correspondió a un estudio de tipo experimental, el cual se realizó durante los meses de marzo a junio del año 2012.

La muestra biológica estuvo constituida por anillos aórticos procedentes de 14 ratas macho, sanas, de la especie Rattus norvegicus, variedad albina swiss, de cuatro meses de edad, con un peso aproximado de 250 a $300 \mathrm{~g}$, criados en el bioterio del Centro de Investigación y Desarrollo Científico (CIDEC) de la Facultad de Medicina de la Universidad Nacional de San Agustín (UNSA), en Arequipa, Perú. Para el manejo de los animales se consideró las normas y procedimientos éticos establecidos internacionalmente en la Guide for the care and use of laboratory animals of the National Research Council, Washington, DC. Se les dio alimento de mantenimiento y se las mantuvo con ciclo regular de luz/oscuridad de 12 horas. Las ratas fueron sometidas a muerte por contusión cervical y se les practicó una incisión pre esternal, abordando su cavidad torácica. Se procedió a remover la aorta torácica, eliminando la adventicia y el tejido conectivo periaórtico. Se seccionó las aortas en anillos de 5 mm de longitud; de cada aorta se obtuvo 5 anillos y cada anillo fue utilizado en experimentos diferentes.

Se preparó el extracto hidroalcohólico usando hojas de Olea europaea (olivo) provenientes de un cultivo orgánico maduro de la región Arequipa (2 $340 \mathrm{msnm}$ ). El registro taxonómico fue hecho por la Facultad de Biología de la UNSA. La preparación se hizo siguiendo el procedimiento descrito por Arroyo y col ${ }^{(17)}$. Se las limpió y dejó secar a temperatura ambiente, protegidas de la luz durante tres semanas; posteriormente, fueron pulverizadas en molino de granos y se agregó etanol al $96 \%$, en una proporción de $150 \mathrm{~mL}$ de alcohol más $50 \mathrm{~mL}$ de agua por cada $100 \mathrm{~g}$ de pulverizado y se dejó macerar por 8 días con protección de la luz. La solución restante fue filtrada y se dejó evaporando en baño maría a $37^{\circ} \mathrm{C}$ por 24 horas. El producto que se obtuvo se guardó en ambiente seco y protegido de la luz solar.

En la preparación de los anillos aórticos y determinación de los patrones de contracción y relajación, en cada prueba se utilizó el método descrito por Furchgott y col ${ }^{(18,19)}$. Los anillos fueron insertados dentro de una cámara para órgano aislado, mediante dos ganchos de acero inoxidable que lo sujetaban, uno de ellos fijo e insertado en el borde de la cámara y el otro conectado a un transductor de tensión isométrica y a un polígrafo Grass. Se llenó el pozo de la cámara con $800 \mu \mathrm{l}$ de solución Krebs-Henseleit compuesta por $\mathrm{NaCl}$, $\mathrm{KCl}, \mathrm{KH}_{2} \mathrm{PO}_{4}, \mathrm{SO}_{4} \mathrm{Mg}, \mathrm{CaCl}_{2}, \mathrm{NaHCO}_{3}$ y glucosa; fue mantenida con burbujeo continuo de una mezcla carbógena de gases $\left(95 \%\right.$ de $\mathrm{O}_{2}$ y $5 \%$ de $\left.\mathrm{CO}_{2}\right)$. La temperatura de la solución dentro de la cámara se mantuvo a $37,0 \pm 0,5^{\circ} \mathrm{C}$, con el empleo de un termociclador y termostato. Se aplicó una tensión equivalente a $120 \mathrm{mmHg}$, mediante su estiramiento con una pesa de $2 \mathrm{~g}$, y se dejó reposar por 60 minutos aproximadamente con recambio de la solución Krebs-Henseleit cada 10 minutos, para lograr su estabilización. Luego, fue necesario contraer los anillos aórticos con una solución de $\mathrm{CaCl}_{2} 6 \mathrm{mM}$, para después estimular su relajación con acetilcolina (ACh) $10 \mu \mathrm{M}$ y así comprobar el estado funcional de cada anillo. Para cada caso se midió la altura en milímetros de la curva graficada por el polígrafo Grass para la contracción máxima alcanzada y se la designó arbitrariamente como el 100\% de vasoconstricción. 
Para evaluar el efecto vasodilatador, los anillos fueron contraídos, agregándose al baño $\mathrm{CaCl}_{2} 6 \mathrm{mM}$, para obtener contracción máxima sostenida, y luego se agregó el extracto hidroalcohólico de Olea europaea en tres dosis: $25 \mathrm{mg} / \mathrm{mL}$, $50 \mathrm{mg} / \mathrm{mL}, 100 \mathrm{mg} / \mathrm{mL}$, registrándose la actividad vasomotora durante $10 \mathrm{mi}$ nutos. Se realizó 10 pruebas para cada concentración con anillos aórticos diferentes y luego de medir las alturas en milímetros de las curvas graficadas, se calculó el porcentaje de vasodilatación, considerando la máxima contracción sostenida de cada caso como el $100 \%$. Previamente a cada experimento se empleó como control en cada anillo una solución hidroalcohólica al 70\% sin extracto de hoja de Olea europaea, para descartar algún efecto vasodilatador del alcohol.

Para determinar el mecanismo fisiológico del efecto vasodilatador, a un grupo de 10 anillos se le aplicó extracto hidroalcohólico de hoja de Olea europaea $100 \mathrm{mg} / \mathrm{mL}$ después de generar vasoconstricción sostenida con $\mathrm{CaCl}_{2}$ $6 \mathrm{mM}$ y se observó la motilidad por 10 minutos. Luego se agregó $1 \mathrm{H}-[1,2,4]$ oxadiazolo [4,3-alquinoxalin-1-one] (ODQ) $100 \mathrm{mM}$, que es un inhibidor de la enzima guanilatociclasa, y nuevamente se observó la vasomotilidad por 10 minutos. El propósito fue bloquear la parte final de la vía endotelial, es decir, la producción de GMP cíclico en el músculo liso vascular, y evaluar la repercusión sobre el efecto vasodilatador. Otro grupo de 10 anillos fue incubado por 20 minutos en ODQ 100 $\mathrm{mM}$ seguido de otros 20 minutos en nifedipino $5 \mu \mathrm{M}$ (fármaco inhibidor de los canales de calcio del músculo liso vascular). Posteriormente, se generó vasoconstricción sostenida con $\mathrm{CaCl}_{2}$ $6 \mathrm{mM}$ y después se administró extracto a una dosis de $100 \mathrm{mg} / \mathrm{mL}$, observando la vasomotilidad por 10 minutos. Esto se hizo para evaluar la repercusión sobre el efecto vasodilatador del extracto tras bloquear los canales de calcio. Se aplicó una solución hidroalcohólica al $70 \%$ sin extracto como control en cada uno de los experimentos. Con la altura en milímetros de la curva graficada se calculó el porcentaje de vasodilatación respecto a la contracción máxima.

La inhibición de la vasoconstricción se evaluó determinando la máxima contracción sostenida de 20 anillos tras la administración de $\mathrm{CaCl}_{2}$. Luego se incubó 10 anillos en captopril $10 \mu \mathrm{M}$ y los otros 10 anillos en extracto hidroalcohólico de hoja de Olea europaea 100 $\mathrm{mg} / \mathrm{mL}$. En ambos casos, la incubación fue por 30 minutos. A continuación se procedió a generar nuevamente vasoconstricción con $\mathrm{CaCl}_{2} 6 \mathrm{mM}$ en ambos grupos. Se calculó el porcentaje de contracción, correspondiente a la altura en milímetros de la curva graficada por el polígrafo, respecto a la contracción máxima (100\%). Se comparó en cada caso el porcentaje de contracción máxima con el porcentaje de contracción posterior a la incubación. Previamente a cada prueba con extracto se utilizó como control en cada anillo la incubación en una solución hidroalcohólica al 70\% sin extracto, para descartar algún efecto provocado por los solventes. Se evaluó el efecto inhibidor de la vasoconstricción por ser un probable efecto, alternativo a la vasodilatación, por el cual el extracto de hoja de olivo presentaba su actividad hipotensora.

En total, se obtuvo 70 anillos aórticos (5 anillos de cada una de las 14 ratas) para las evaluaciones del efecto vasodilatador, la inhibición de la vasoconstricción y, además, la determinación del mecanismo fisiológico de la vasodilatación. Al ser un trabajo experimental, se utilizó para todos los casos solución hidroalcohólica al 70\% en anillos aórticos como grupo control. Cada anillo se usó solamente en dos oportunidades, control y experimento, luego del cual fue descartado; para cada nuevo experimento se utilizó un anillo aórtico intacto.

La sustancia vasoconstrictora empleada fue el cloruro de calcio $\left(\mathrm{CaCl}_{2}\right)$, el cual ejerce un efecto vasoconstrictor in vitro por 2 horas como mínimo, tiempo en el cual se desarrollaron los experimentos que duraron en prome- dio 1 hora y media. Las demás sustancias utilizadas -captopril, nifedipino y ODQ- ejercieron una acción efectiva in vitro a partir de los 15 minutos de incubación y tuvieron una duración máxima a los 45 minutos, tiempo en el que se realizó los ensayos.

Para la base de datos y los gráficos se utilizó el programa Microsoft Office Excel 2010. El análisis de los datos se realizó con el paquete estadístico SPSS v.15 s. Se aplicó las pruebas de normalidad de Kolmogorov-Smirnov y el estadístico t student para la prueba de diferencia de medias, con un nivel de significancia de $p<0,05$.

\section{RESULTADOS}

Se obtuvo un patrón de contracción máxima de anillos de aorta de ratas normales tras la administración de una solución de $6 \mathrm{mM}$ de cloruro de calcio $\left(\mathrm{CaCl}_{2}\right)$ y la relajación fisiológica con $10 \mu \mathrm{M}$ de acetilcolina (ACh). En la figura 1 se observa la vasodilatación de los anillos aórticos provocada por el extracto hidroalcohólico de hoja de Olea europaea con las diferentes dosis administradas. Las dosis de 25, 50 y $100 \mathrm{mg} / \mathrm{mL}$ produjeron vasodilatación en un promedio de 7,20 $\pm 1,49 \%, 9,84$ $\pm 1,42 \%$ y $12,31 \pm 1,16 \%$, respectivamente. Existió diferencia significativa con respecto al $100 \%$ de contracción solamente para el caso de la tercera dosis; es decir, para la dosis de $100 \mathrm{mg} /$ $\mathrm{mL}$ ( $\mathrm{t}$ student para grupos pareados, $\mathrm{p}$ $<0,05)$.

Se observó un incremento del efecto vasodilatador del extracto hidroalcohólico de hoja de Olea europaea a una concentración de $100 \mathrm{mg} / \mathrm{mL}$ después de administrar $1 \mathrm{H}-[1,2,4]$ oxadiazolo [4,3-alquinoxalin-1-one] (ODQ) 10 $\mathrm{mM}$. El efecto vasodilatador aumentó significativamente a un promedio de $22,93 \pm 5,32 \%$, resultando diferente de la máxima contracción sostenida o $100 \%$ (t student para grupos pareados, $\mathrm{p}<0,05)$.

El efecto vasodilatador del extracto, luego de la incubación de los 
anillos aórticos en ODQ $10 \mathrm{mM}$ y nifedipino $5 \mu \mathrm{M}$ por 20 minutos en cada uno, se redujo a un promedio de $2,62 \pm 1,12 \%$, pero no fue diferente con respecto al $100 \%$ de contracción, por lo que no se consideró vasodilatación. Se halló por tanto una reducción del efecto vasodilatador, lo que sugiere que el principal mecanismo por el que se genera la vasodilatación es una inhibición de los canales de calcio del músculo liso vascular.

La contracción máxima de los anillos aórticos con cloruro de calcio, después de la incubación por 30 minutos en extracto hidroalcohólico de hoja de Olea europaea a $100 \mathrm{mg} / \mathrm{mL}$ y en captopril $10 \mu \mathrm{M}$, se redujo a un promedio de $72,0 \pm 1,57 \%$ y $74,49 \pm 2,90 \%$ para el caso de la incubación en extracto y en captopril, respectivamente. No se encontró diferencia significativa entre ambos resultados, pero sí de cada uno con respecto al 100\% de contracción.

\section{DISCUSIÓN}

El patrón de contracción máxima obtenido demuestra que los anillos aórticos utilizados se encontraban en perfectas condiciones para poder realizar los experimentos posteriores. El efecto vasodilatador provocado por el extracto hidroalcohólico de Olea europaea fue en aumento conforme se incrementó la dosis hasta $100 \mathrm{mg} / \mathrm{mL}$ (figura 1), es decir, sigue un patrón dependiente de la dosis, que sugiere la posible participación de una vía enzimática, pues sigue una curva cinética similar a la de estas. Únicamente la respuesta obtenida con la administración de $100 \mathrm{mg} / \mathrm{mL}$ de extracto resultó diferente de la máxima contracción sostenida $(\mathrm{p}<0,05)$. Por lo tanto, se considera que provocó vasodilatación. Lo anterior concuerda con lo señalado por Zarzuelo A y col ${ }^{(16)}$, quienes también encontraron el mismo efecto vasodilatador provocado por las hojas de olivo, aunque con concentraciones diferentes; esto posiblemente por varias razones, dentro de las que podemos mencionar el uso de hojas de otra variedad de olivo o el mayor grado

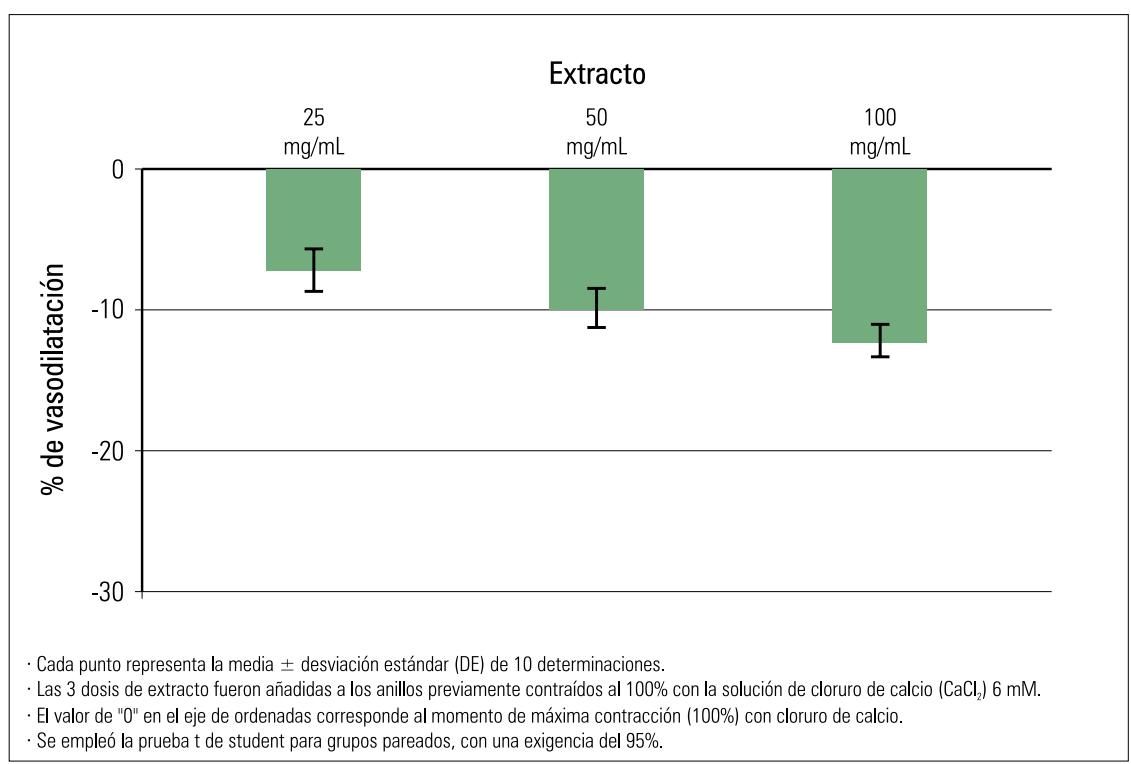

Figura 1. Efecto vasodilatador del extracto hidroalcohólico de hoja de Olea europaea en anillos aórticos de rata a 3 dosis crecientes $(25,50$ y $100 \mathrm{mg} / \mathrm{mL})$ con respecto a la contracción máxima (100\%) con $\mathrm{CaCl}_{2}$.

de pureza obtenido en los procesos de extracción. El efecto vasodilatador provocado por el extracto probablemente sea debido al contenido abundante de compuestos fenólicos, como la oleuropeina y el hidroxitirosol; este último también abunda en el fruto de Olea europea y es señalado como el principal responsable de las propiedades terapéuticas que presenta el olivo. Numerosos estudios anteriores, como el realizado por P. de la Fuente y col (2), han demostrado que el hidroxitirosol tiene una enorme capacidad antioxidante, antimicrobiana, antiesclerótica y hasta apoptótica. Dichas propiedades serían consecuencia de su capacidad para modular distintos procesos enzimáticos, algunos de los cuales son importantes para controlar el tono vascular ${ }^{(20,21)}$.

Se observó un marcado aumento de la vasodilatación provocada con extracto a $100 \mathrm{mg} / \mathrm{mL}$ después de la administración de ODQ $100 \mathrm{mM}$, que resultó significativo $(p<0,05)$. La fase final de la vía endotelial se lleva a cabo cuando el óxido nítrico provoca la formación de GMP cíclico por la enzima guanilatociclasa en el músculo liso vascular, para posteriormente, a través de este, desencadenar los mecanismos intracelulares que lleven a la relajación de los vasos sanguíneos. La persistencia del efecto vasodilatador ante el bloqueo de la producción de GMP cíclico provocado al agregar el ODQ indicaría la independencia de la vía endotelial o en todo caso la mínima intervención de esta vía en la producción de dicho efecto; caso contrario, se habría manifestado una inhibición de la vasodilatación. Esta observación concuerda con lo descrito por Gilani AH y col ${ }^{(22)}$, quienes proponen que la respuesta vasodilatadora del olivo y sus hojas sería provocado por una acción sobre los canales de calcio presentes en la membrana celular de las células musculares lisas de los vasos sanguíneos.

La inhibición de la producción de GMP cíclico y el bloqueo conjunto de los canales de calcio obtenido con la incubación previa de los anillos en ODQ y nifedipino provocó una reducción del efecto vasodilatador del extracto de hojas de Olea europaea; ello sugiere que el principal mecanismo por el que se genera la vasodilatación es una inhibición de los canales de calcio del músculo liso vascular. Este resultado es semejante al de estudios realizados por Gilani AH y col (22) y Scheffler A y col ${ }^{(23)}$, quienes 
señalan que el extracto actuaría como un antagonista de los canales mencionados, reduciendo así la entrada de calcio del extracelular a las células musculares; ello disminuye la concentración de calcio intracelular y provocaría vasodilatación. Sin embargo, pese a los resultados obtenidos no se descarta la parcial intervención sobre la vía endotelial, en vista de que en el estudio de Khayyal MT y col (24) se observó un efecto hipotensor luego de la administración de un extracto de hoja de Olea europea a ratas con hipertensión inducida con L-NAME, una sustancia que inactiva la vía endotelial.

El nifedipino actúa inhibiendo los canales de calcio del tipo L (canales lentos) ${ }^{(25)}$. Esta acción redujo el efecto vasodilatador sobre los anillos aórticos, pues se habría bloqueado el sitio de acción del extracto y ello indica que estaría actuando sobre el mismo tipo de canales. El extracto hidroalcohólico de hojas Olea europaea muestra así una acción comparable con la del nifedipino, lo que resulta de mucha importancia, pues resalta la necesidad de iniciar estudios orientados a la posibilidad de emplear el extracto para otros usos clínicos, además de antihipertensivo, en los que se suele administrar nifedipino, como en el caso de la enfermedad de Raynaud, arritmias cardiacas y para bloquear las contracciones uterinas pretérmino en el trabajo de parto prematuro ${ }^{(25)} \mathrm{y}$, posiblemente, con menores efectos adversos, por tratarse de un derivado natural.

La incubación con extracto hidroalcohólico de Olea europaea provocó la inhibición de la vasoconstricción en los anillos aórticos de rata y resultó significativa al compararla con el patrón de máxima contracción (figura 2). Por otro lado, no hubo diferencia significativa entre la inhibición de la vasoconstricción lograda con el extracto y la obtenida con captopril ( $p>0,05)$, por lo que su efecto es comparable con el de este fármaco. Existen estudios recientes que corresponden con este resultado, como el realizado por Susalit E y col ${ }^{(26)}$, que demuestran igual eficacia hipotensora entre el captopril y el extracto de hoja de olivo al administrarlo en pacientes hipertensos, lo que incrementa la evidencia de que el extracto de hoja

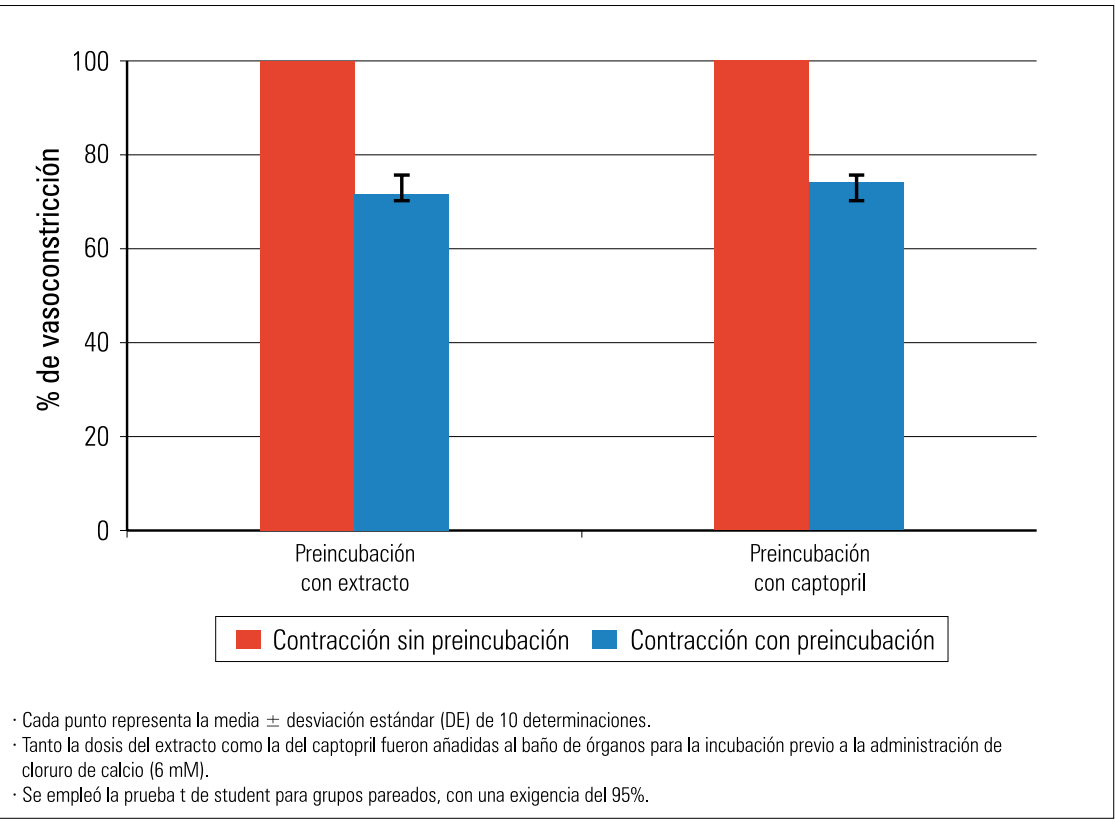

Figura 2. Efecto sobre la respuesta patrón al cloruro de calcio $\left(\mathrm{CaCl}_{2}\right)$ de anillos aórticos normales previamente incubados con extracto hidroalcohólico de Olea europaea $(100 \mathrm{mg} / \mathrm{mL})$ o con captopril $(10 \mu \mathrm{M})$. de olivo también actúa inhibiendo la vasoconstricción. En base a ello, este efecto inhibidor de vasoconstricción se produciría por el mismo mecanismo de acción del captopril, es decir, la inhibición de la enzima convertidora de angiotensina (ECA). Existe respaldo en este resultado en base al estudio de Loizzo MR ${ }^{(27)}$, quien demostró que los compuestos fenólicos contenidos en el olivo presentan in vitro actividad inhibidora de la ECA y destaca nuevamente la participación del hidroxitirosol además de otros compuestos fenólicos.

La existencia de este segundo efecto manifestado por el extracto resulta de mucho interés, porque incrementa la posibilidad de una futura terapia antihipertensiva alternativa. Identificar los componentes del extracto que pertenecen al grupo de antihipertensivos propuesto e iniciar estudios clínicos con ellos incrementará la trascendencia, ya que actualmente cada vez son más los partidarios del empleo de los inhibidores de la ECA como tratamiento de primera línea contra la hipertensión, en vista de que poseen menos efectos adversos ${ }^{(28)}$.

La actividad hipotensora del fruto y del extracto de hoja de Olea europaea ha sido demostrada en otros estudios anteriores y sin duda los efectos vasodilatador e inhibidor de la vasoconstricción demostrados en el presente estudio están entre los principales responsables de la manifestación de dicha actividad. A pesar de los resultados obtenidos no podemos extrapolar estos en su totalidad al ser humano, ni reemplazar la terapia farmacológica, ya que se hacen necesarios estudios posteriores, partiendo por aislar del extracto los componentes involucrados, seguido de aspectos farmacocinéticos y posológicos. Sin embargo, los resultados obtenidos apuntan a reconocer el gran valor de las hojas de olivo y un futuro prometedor en la búsqueda de mejores alternativas para la terapia antihipertensiva.

En conclusión, el extracto hidroalcohólico de hojas de Olea europaea, en dosis de $100 \mathrm{mg} / \mathrm{mL}$ tuvo un efecto 
vasodilatador sobre anillos aórticos de rata mediado por el bloqueo de canales de calcio y además presentó un efecto inhibidor de la vasoconstricción.

Recomendamos realizar otros estudios con el fin de definir si el hidroxitirosol también es responsable directo de estos efectos demostrados. Además, sugerimos estudios posteriores para evaluar el efecto vasodilatador del extracto sobre vasos sanguíneos con disfunción endotelial y la aplicación sobre enfermedades relacionadas.

\section{AGRADECIMIENTOS}

A nuestros asesores. Mg. Narendra Vera Núñez, Dr. Javier Rojas Ticona y de manera especial al Dr. Azael Paz Aliaga, Jefe del Departamento de Ciencias Fisiológicas de la Facultad de Medicina de la Universidad Nacional de San Agustín y Director del Centro de Investigación y Desarrollo Científico de la misma universidad. A nuestros colaboradores Lic. Manuel Pariguana Begazo, Sr. Rafael Loaiza, Sr. Otto Núñez, Srta. Margaret Paola Lipa Ale, Srta. Carmen Chávez Chávez.

\section{REFERENCIAS BIBLIOGRÁFICAS}

1. Font Quer P. Plantas medicinales el Dioscórides re-

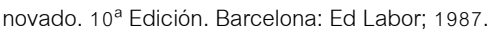

2. de la Fuente P, Chamorro P, Moreno M, Poza MA. Propiedades antioxidantes del hidroxitirosol procedente de la hoja de olivo (Olea europaea L.). Rev Fitoterapia. 2004;4(2):139-47.

3. EI SN, Karakaya S. Olive tree (Olea europaea) leaves: potential beneficial effects on human health. Nutr Rev. 2009;67(11):632-8.

4. Briante R, Patumi M, Terenziani S, Bismuto E, Febbraio F, Nucci R. Olea europaea L leaf extract and derivatives: antioxidant properties. J Agric Food Chem. 2002;50:4934-40.
5. Ghanbari R, Anwar F, Alkharfy KM, Gilani A-H, Saari $N$. Valuable nutrients and functional bioactives in different parts of olive (Olea europaea L.). A review. Int J Mol Ciencia. 2012;13(3):3291-340.

6. Ryan D, Prenzler PD, Lavee S, Antolovich M, Robards K. Quantitative changes in phenolic content during physiological development of the olive (Olea europaea) cultivar Hardy's Mammoth. J Agr Food Chem. 2003;51:2532-8.

7. Togna GI, Togna AR, Franconi M, Marra C, Guiso M. Olive oil isochromans inhibit human platelet reactivity. J Nutr. 2003;133(8):2532-6.

8. Al-Azzawi HF, Alhamdani MS. Hypoglycemic and antioxidant effect of oleuropein in alloxan-diabetic rabbits. Life Sci. 2006;78(12):1371-7.

9. Vissers MN, Zock PL, Katan MB. Bioavailability and antioxidant effects of olive oil phenols in humans: a review. Eur J Clin Nutr. 2004;58(6):955-65.

10. Samuelsson $\mathrm{G}$. The blood pressure lowering factor in leaves of Olea europaea. Farmacevtisk Revy. 1951;15:229-39.

11. Agusti RC. Epidemiologia de la hipertensión arterial en el Perú. Acta Med Peru. 2006;23(2):69-75.

12. Khayyal MT, el-Ghazaly MA, Abdallah DM, Nassar NN, Okpanyi SN, Kreuter MH. Blood pressure lowering effect of an olive leaf extract (Olea europaea) in L-NAME induced hypertension in rats. Arzneimittelforschung. 2002;52(11):797-802.

13. Somova LI, Shode FO, Ramnanan P, Nadar A. Antihypertensive, antiatherosclerotic and antioxidant activity of triterpenoids isolated from Oleaeuropaea, subspecies africana leaves. J Ethnopharmacol. 2003;84(2-3):299-305.

14. Cherif S, Rahal N, Haouala M, Hizaoui B, Dargouth F, Gueddiche M, Kallel Z, Balansard G, Boukef K. A clinical trial of a titrated Olea extract in the treatment of essential arterial hypertension. J Pharm Belg. 1996;51(2):69-71.

15. Perrinjaquet-Moccetti $T$, Busjahn A, Schmidlin C, Schmidt A, Bradl B, Aydogan C. Food supplementation with an olive (Olea europaea L.) leaf extract reduces blood pressure in borderline hypertensive monozygotic twins. Phytother Res. 2008;22(9):1239-42.

16. Zarzuelo A, Duarte J, Jimenez J, González M, Utrilla MP. Vasodilator effect of olive leaf. Planta Med. 1991;57:417-9.

17. Arroyo J, Ráez E, Rodríguez M, Chumpitaz V, Burga J, De la Cruz W, et al. Actividad antihipertensiva y antioxidante del extracto hidroalcohólico atomizado de maiz morado (Zea mays L.) en ratas. Rev Peru Med Exp Salud Publica. 2008;25(2):195-9.

18. Furchgott RF, Zawadzki JV. The obligatory role of endothelial cells in the relaxation of arterial smooth muscle by acetylcholine. Nature. 1980;288(5789):373-6.

19. Furchgott RF, Vanhoutte PM. Endotheliumderived relaxing and contracting factors. FASEB J. 1989;3(9):2007-18.
20. Covas MI, de la Torre K, Farré-Albaladejo M, Kaikkonen J, Fitó M, López-Sabater C, et al. Postprandial LDL phenolic content and LDL oxidation are modulated by olive oil phenolic compounds in humans. Free Radic Biol Med. 2006;40(4):608-16.

21. Covas MI, Nyyssonen K, Poulsen HE, Zunft $\mathrm{H}-\mathrm{JF}$, Kiesewetter H, Gaddi A, et al. The effect of polyphenols in olive oil on heart disease risk factors: a randomized trial. Ann Intern Med. 2006;145(5):333-41.

22. Gilani $A H$, Khan $A U$, Shah AJ, Connor J, Jabeen $Q$. Blood pressure lowering effect of olive is mediated through calcium channel blockade. Int J Food SciNutr. 2005;56:613-20.

23. Scheffler A, Rauwald HW, Kampa B, Mann U, Mohr FW, Dhein S. Olea europaea leaf extract exerts L-Type $\mathrm{Ca}(2+)$ channel antagonistic effects. J Ethnopharmacol. 2008;120:233-40.

24. Khayyal MT, el-Ghazaly MA, Abdallah DM, Nassar NN, Okpanyi SN, Kreuter MH. Blood pressure lowering effect of an olive leaf extract (Olea europaea) in L-NAME induced hypertension in rats. Arzneimittelforschung. 2002;52(11):797-802.

25. Goodman \& Gilman. Las bases farmacológicas de la terapéutica. 12a Edición. McGraw-Hill Interamericana Editores; 2012.

26. Susalit E, Agus N, Effendi I, Tjandrawinata RR, Nofiarny D, Moccetti T-P, Verbruggen M. Olive (Olea europaea) leaf extract effective in patients with stage-1 hypertension: comparison with Captopril. Fitomedicina. 2011;18(4):251-8.

27. Loizzo M R, Lecce G D, Boselli E, Menichini F, Frega N G. Inhibitory activity of phenolic compounds from extra virgin olive oils on the enzymes involved in diabetes, obesity and hypertension. J Food Biochem. 2011;35:381-99.

28. Kasper DL, Braunwald E, Fauci AS, Hauser SL, Longo DL, Jameson JL. Harrison's Principles of Internal Medicine. 17a Edición. United States of America: McGraw-Hill Interamericana Editores; 2008.

Artículo recibido el 15 de mayo de 2013 y aceptado para publicación el 23 de mayo de 2013.

Fuente de financiación: Estudio autofinanciado.

Conflicto de intrereses: No existen en el presente trabajo.

Correspondencia:

Job Nexar Quispe Huamán

Urb. San Salvador E-1 Paucarpata, Arequipa, Perú. Correo electrónico: an_aysjobnex_tj7@hotmail.com 